\title{
Multiband Array Antenna towards Microstrip Patch antenna using Resonant Frequency for s band Application
}

\author{
Nivedita Mishra \\ Department of Electronics and \\ communication Engineering \\ Integral university, Lucknow \\ nivimini01@gmail.com
}

\author{
Dr Saima Beg \\ Department of Electronics and \\ communication Engineering \\ Integral university, Lucknow \\ saimabeg@iul.ac.in
}

\author{
Preeti Singh \\ Department of Electronics and \\ communication Engineering \\ UIET, CSJMU, Kanpur \\ preeti.12kec@gmail.com
}

\begin{abstract}
In today's world, requirement for lower-weight, low-cost, low-profile, and effective-use transmitters is growing by the day. For addressing these parameters in a variety of applications, microstrip patch antennas are more popular than other antennas. The main issue with these types of microstirp patch antennas is that they have a high return loss, VSWR, and bandwidth, but these issues can be rectified utilising a design technique. The method of design was to use mirror image layouts, which was a simple and easy way to improve performance. The efficiency of a simple patch cannot be influenced by any single structure that lacks a mirror reflection. In this study, the two +shaped constructions are used, and the results are in good agreement. In today's world, these antennas have a variety of uses, including WLAN and $s$ band applications. In future, various frameworks will be used to improve the results that have been produced thus far.
\end{abstract}

Keywords- Bandwidth, Rectangular Microstrip patch Antenna(RMPA), Return Loss, Ground Plane, FR4 ,Resonant frequency.

1.Introduction In recent years, advancements in communications networks have necessitated the creation of low-cost, light-weight, low-profile antennas capable of delivering great performance across a broad frequency band. The goal of prospective personal communication device development will be to allow image, voice, and data communications at any moment and from everywhere on the planet. To adequately cover the available operating bands, new communication endpoint transmitters must meet criteria of multi-band or wideband activities. The manufactured transmitter performance was measured and matched to modeling findings. In addition, we've discussed how to use FR4 for a variety of objectives, including antenna area diminution and other mode alteration-related tasks. The efficiency of rectangular patch transmitter built on FR4 substrate has been compared to that of similar antenna built on a standard FR4 substrate. Microstrip patch antennas are extensively employed in wireless devices in modern wireless communication networks. As a result, transmitter shrinking has become a critical challenge in lowering the overall size of the communication system. Microstrip patch antennas are extensively employed in wireless devices in contemporary wireless communication networks. The suggested Antenna's advantages, such as light weight, lower profile, lower cost, easy integration with innovation and engineering, and considerably simple production, are driving demand in commercial and military wireless systems. All of these antennas may be manufactured with the help of IE3D simulation software and have extremely crisp features. Because of its lower profile and tiny size, suggested RMPA can be employed in a wide range of wireless communication technologies. Due to its lower profile and smaller weight, microstrip antennas are widely utilized in radio communication technologies A Microstrip, Patch transmitter in its most basic form, contains a transmitting patch on one end of dielectric substrate with a grounding plane on other. The patch is typically made of conductive metals like gold or copper and may be manufactured into any shape. The transmitting patch and feed lines is normally photo etched on 
dielectric substrate. The impedance bandwidth and buy back loss are critical factors of any form of antenna. The impedance bandwidth is determined by the specifications of the patch transmitter component and the feed utilized. The bandwidth is usually restricted to fewer percent of the total. This is one of the drawbacks of a standard patch transmitter. The bandwidth and transmission loss of a FR4-based rectangular microstrip patch transmitter are significantly improved. The FR4-based rectangular microstrip patch antenna was designed using IE3D, a software tool for electromagnetic analysis and design.

\section{THE SUGGESTED ANTENNA STRUCTURE'S DESIGN METHODOLOGY AND} SIMULATION

A design approach is detailed based on the reduced formulation that leads to the realistic development of square microstrip patch antennas. The process assumes that given information comprises the substrate's dielectric constant (r), resonant frequency (fr), substrate height (h), and loss tangent. $(\delta)$. SPECIFY :- Er $=4.4$

$\mathrm{h}=1.6 \mathrm{~mm}$

$\mathrm{fc}=2 \mathrm{GHz} 2$.

$\delta=0.02$

Zeland Inc's IE3D was used to design and test the Microstrip patch transmitter. The electromagnetic simulation IE3D is built on the method of moments and is a full electromagnetic simulation. It examines 3D and multilayer general form structures. Patch, transmitters wire antennas, and other $\mathrm{RF} /$ wireless antennas have all been designed with it. It may be utilized to determine the return loss, VSWR, and bandwidth.

The following are the critical criteria for designing rectangular microstrip Patch Antenna:

- Length(L): Two sides were chosen to be $45.64 \mathrm{~mm}$ in length each.

- Width(W): Two sides were chosen to be $35.14 \mathrm{~mm}$ in length each.

- Frequency of operation(fo): The transmitters' frequency response should be chosen carefully. For our design, we chose a resonance frequency of $2.0 \mathrm{GHz}$.

- Dielectric constant of substrate(Er): The dielectric constant of the material we chose for theor design is 4.40. Because it lowers the antenna's dimensions, a substrate with just a large dielectric constant was chosen.

- Height of dielectric material (h): It is critical that the microstrip patch antennas used in cellular phones are not too bulky. As a result, the dielectric substrate has a height of $1.6 \mathrm{~mm}$.

- Length (L) and Cutting Width (w): $5.0 \mathrm{~mm}$ and $10.0 \mathrm{~mm}$ were chosen as the length and width, respectively

Hence, the design's most important aspects are:

$\begin{array}{lll}\mathrm{L} & = & 45.64 \mathrm{~mm} \\ \mathrm{~W} & = & 35.14 \mathrm{~mm} \\ \text { Cutting Width } & = & 5.0 \mathrm{~mm} \\ \text { Cutting Length } & = & 10.0 \mathrm{~mm} \\ \text { fo } & = & 2.0 \mathrm{GHz} \\ \mathrm{\varepsilon r} & = & 4.40 \\ \mathrm{~h} & = & 1.60 \mathrm{~mm} \\ \text { Tangent loss } & = & 0.02\end{array}$




\subsection{Designing Microstrip Patch Antenna}

\section{Step \# 01: Computation of the Width $(W)$}

The Microstrip patch antenna's width is specified as:

$$
\frac{1}{2 F r \sqrt{(\mu \varepsilon)}} \sqrt{\left(\frac{2}{(\varepsilon r+1)}\right)}=\frac{C}{2 F r} \sqrt{\left(\frac{2}{(\varepsilon r+1)}\right)}
$$

Replacing c $=3.00 \mathrm{e}+008 \mathrm{~m} / \mathrm{sec} ., \varepsilon_{\mathrm{r}}=4.40$ and fo $=2.0 \mathrm{GHz}$

Step \# 02: Computation of Effective dielectric constant (Ereff):

The effectual dielectric constant is:

$$
\varepsilon f f=\frac{\varepsilon r+1}{2}+\frac{\varepsilon r-1}{2}\left(\frac{1}{\sqrt{1+\frac{12 h}{w}}}\right)
$$

Step \# 03: Computation of the Effective length ( Leff)

The effectual length is:

$$
L_{e f f}=\frac{C}{2 f_{0} \sqrt{\varepsilon_{\text {reff }}}}
$$

Replacing Eeff $=4.40, \mathrm{c}=3.00 \mathrm{e}+008 \mathrm{~m} / \mathrm{sec}$ and fo $=2.0 \mathrm{GHz}$

Step \# 04: Computation of the length extension $(\Delta L)$ :

$$
\Delta L=0.412 h \frac{\left(\varepsilon_{r e f} f+0.3\right)\left(\frac{W}{h}+0.264\right)}{\left(\varepsilon_{r e f f}-0.258\right)\left(\frac{W}{h}+0.8\right)}
$$

Step \# 05: Computation of actual length of patch $(L)$ :

The genuine length is produced by:

\section{Step \# 06: Computation Of VSWR}

$$
V S W R=\left(\frac{1+|\Gamma|}{1-|\Gamma|}\right)
$$

\section{Step \# 07: Computation of Return Loss}

When such an electromagnetic wave reaches a mismatched load or indeed a line interruption while travelling down a transmission system, some of incidence power is redirected back down line. The return loss is calculated as follows:

$$
R L=10 \log _{10}(\Gamma) d B
$$

\subsection{Methodology BASED DISIGN}

\begin{tabular}{|c|}
\hline Literature study \\
Volume 23, Issue 7, Ju1. \\
$\begin{array}{c}\text { The parameters that are use in } \varepsilon_{\mathrm{r}}=4.40 \text {, tangent } \\
\text { loss=0.020, h=1.60for simple patch \&3.2 for } \\
\text { modified patch. }\end{array}$ \\
\hline
\end{tabular}




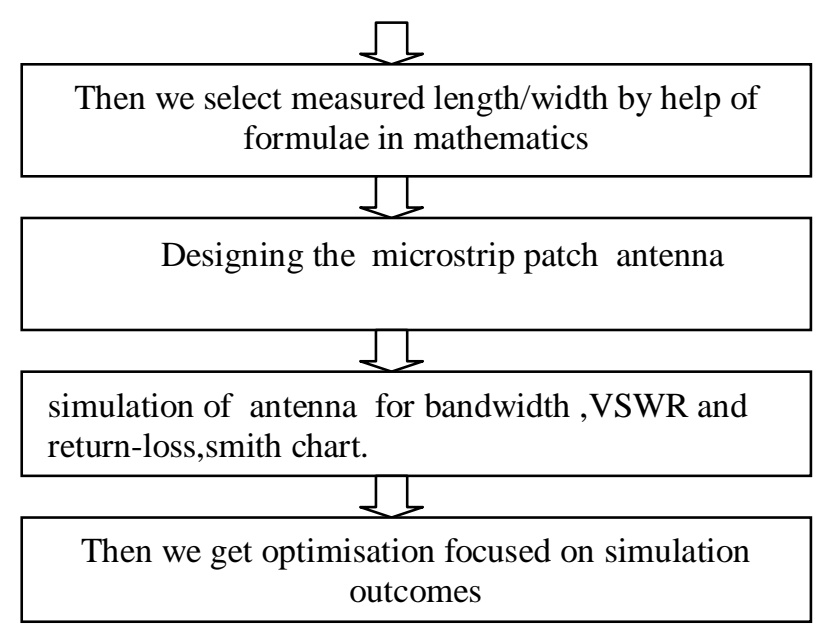

\section{Designingof Rectangular microstrip patch antenna Configuration}

Figure 1 depicts the planned antenna design. The suggested L\&W size is printed on substrate with a height of $h=1.60$ and a comparative permeability of 4.4. On a ground board, the print is scratched. A 50-microstrip line feeds the slot. Figure 1 shows the basic square microstrip line fed printed transmitter design The dimension of is calculated by for stimulating the working frequency at roughly 2 GHz. Figure 1 shows a Basic Rectangular Microstrip Patch Transmitter Feed by Microstrip Line, wherein c is frequency of light in atmosphere, is effectual relative permeability and $\mathrm{L}$ is length. The resonance wavelength of a microstrip patch transmitter is $2 \mathrm{GHz}$. The patch is $35.44 \mathrm{~mm}$ in length and $45.65 \mathrm{~mm}$ in width. The microstrip thread which is utilised for feeding is 32.82mm in length. We used a double-sided copper PCB to create this transmitter. The metal field on lower side of the PCB serves as satellite's ground, and we designed the patch of providing proportions on the upper side of the PCB. To use a dielectric substrate with a altitude of $1.60 \mathrm{~mm}$ and dielectric constant of $4.40 \mathrm{~mm}$, the entire geometry is simulated and raised effectively. The material's loss tangent is 0.02 . The feed to the antenna is provided via microstrip feed line.

\section{Design of simple rectangular microstrip patch antenna}




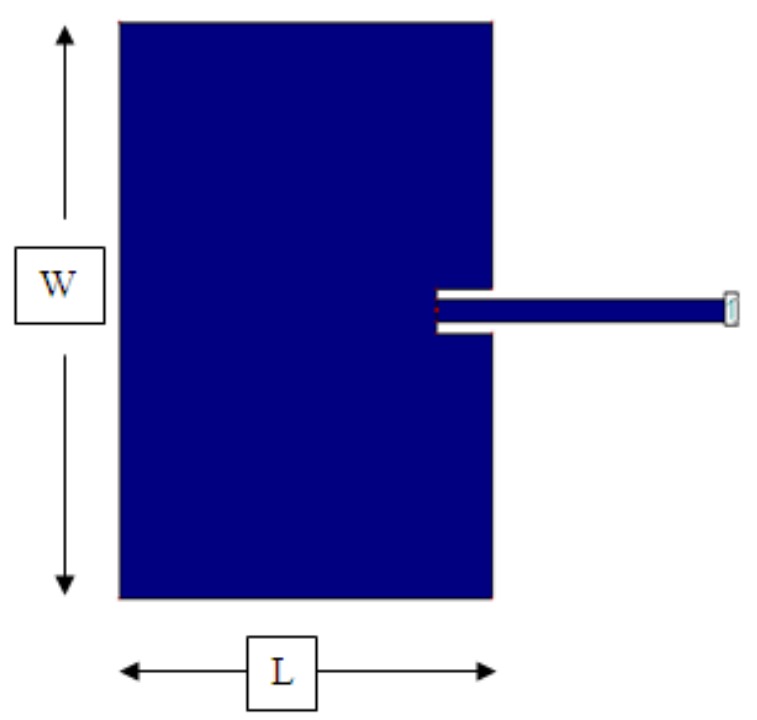

Figure 1(a) Simulated geometry of Simple Patch Antenna

Length of patch antenna $=35.44136 \mathrm{~mm}$

Width of patch antenna $=45.64356 \mathrm{~mm}$

Resonant frequency $=2.0 \mathrm{GHz}$

Summary of Design Parameters of Simple Patch Antenna

\begin{tabular}{|cc|}
\hline Frequency & $2 \mathrm{GHz}$ \\
\hline Length & $35.44 \mathrm{~mm}$ \\
\hline Width & $45.64 \mathrm{~mm}$ \\
\hline Cutwidth & $5.0 \mathrm{~mm}$ \\
\hline Cutdepth & $10.0 \mathrm{~mm}$ \\
\hline Pathlength & $32.82 \mathrm{~mm}$ \\
\hline Pathwidth & $3.009 \mathrm{~mm}$ \\
\hline Return loss & $-26.16 \mathrm{~dB}$ \\
\hline & \\
\hline & 1.132 \\
\hline
\end{tabular}

\subsubsection{S Parameter Display and Bandwidth Calculation}

The simulation is done by varying feeding positions and s-parameter is studied for each simulation and tabulated by taking each case. Thus the enhanced bandwidth of T-shape rectangular microstrip patch is obtained at probe feed position $(1,0)$. 


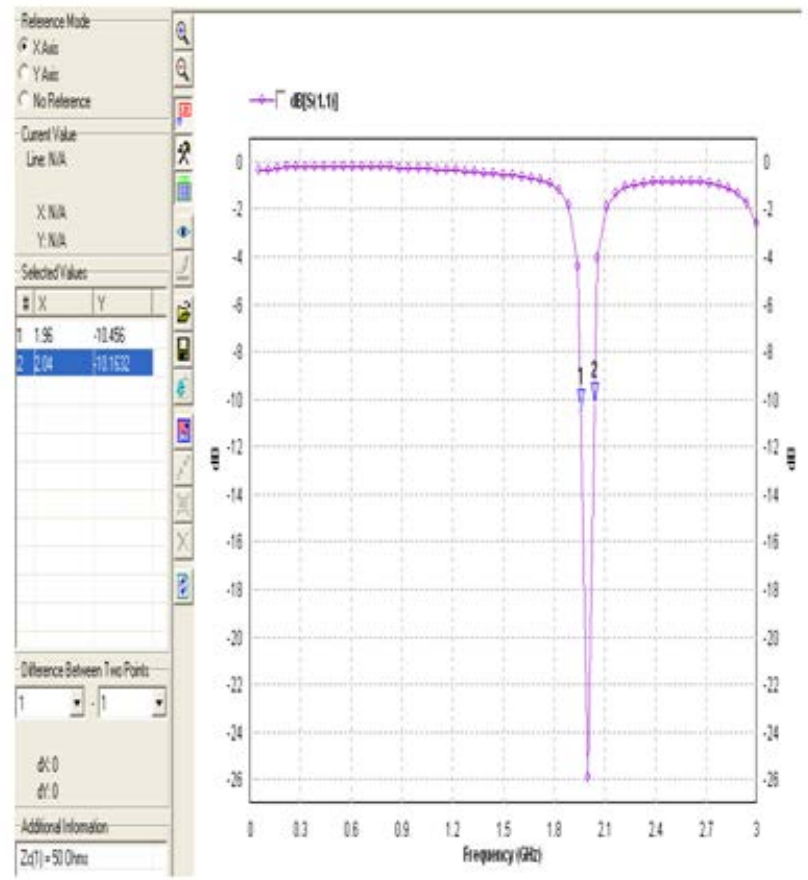

Figure 1(b): S parameter Display

At feed point:(1,0)

$\mathrm{F} 1=1.96 \mathrm{GHz}$

$\mathrm{F} 2=2.04 \mathrm{GHz}$

Bandwidth $=(\mathrm{f} 2-\mathrm{f} 1) * 100 / \mathrm{Fc}$

Bandwidth $=9 \%$

\subsubsection{Bandwidth Computation:}

The bandwidth calculation at feed position $(1,1)$, maximum bandwidth is achieved. From the figure 3.3, frequency $\mathrm{f}_{1}$ is taken as $1.96 \mathrm{GHz}$ and frequency $\mathrm{f} 2$ is taken as $2 \mathrm{GHz}$. Therefore the bandwidth is obtained after doing calculation as shown in figure 3

\subsubsection{Return Loss:}

The return loss at feed position (1,1), we got retrun loss from figure 1(b),the return loss is obtained -26db.

\subsubsection{VSWR:}

We got VSWR form figure 1(c), the VSWR is obtained $>1$. 


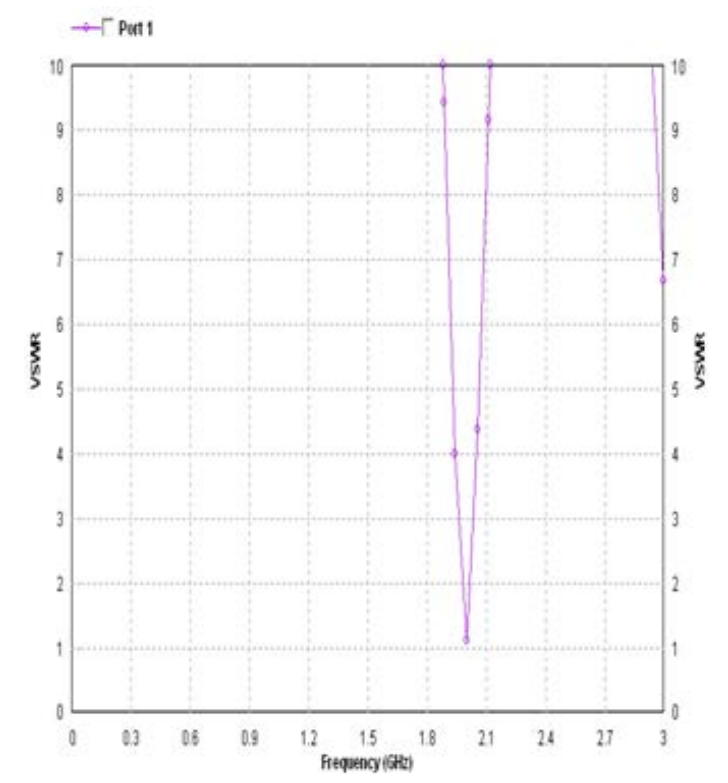

1.(c) VSWR (Voltage Standing Wave Ratio)

\subsection{Geometry \& Designing}

A tiny compact $\mathrm{T}$ shaped microstrip patched transmitter is represented in this paper. To improve the efficiency of this T-shape microstrip patch antenna, reflection designs were used. The performance of a simple patch cannot be influenced by any single structure that lacks a mirror reflection. In this study, the two T-shaped structures are employed, and the results are in strong agreement. The T shape might result in good impedance matching. The proposed microstrip patch satellite's shape is shown in Fig.2. The patch transmitter is developed on a FR4 substrate with a width of $3.20 \mathrm{~mm}$ and a relative dielectric constant (r) of 4.40 , as depicted in Fig.2. It has a small size of $45.64 \mathrm{~mm} \times$ $35.14 \mathrm{~mm}$ (W x L).

A microstrip line with a cut width of $5.0 \mathrm{~mm}$ and a cut depth of $10.0 \mathrm{~mm}$ feeds the radiator. A 50 microstrip line with a ferquancy of $2 \mathrm{GHz}$ is printed on the partially grounded substrate as the excitation.

The modified 1st ground plane controls the impedance bandwidth of a rectangular patch by acting as an impedance matching element. ws stands for antenna width, and ls stands for antenna length.

The suggested antenna may be configured to function at $2.0 \mathrm{GHz}$ frequency by selecting these parameters. The findings of both simulations and experiments are also discussed. This project's simulation results come from Zeland's IE3D simulation.

\section{Design parameters of Modified 1 Microstrip Patch Antenna}

Co-ordinates $1^{\text {st }}$ :

The slot size of

ws1, ws2, ws3, ws4, ws5, ws6, ws7, w8, ws9, ws10, ws11, ls1, ls2, ls3, ls4, ls5, ls6, ls7, ls8, ls9, ls10, ls11 are 10, 10, -10, $-10,-5,-5,-2.5,-2.5,2.5,2.5,5,5,10,7.8,22.8,22.8,7.8,7.8,17.8$, $17.8,7.8,7.8,17.8,17,8,7.8$ and $7.8 \mathrm{~mm}$ respectively. 
Co-ordinates $2^{\text {nd }}$

The slot size of

ws1, ws2, ws3, ws4, ws5, ws6, ws7, w8, ws9, ws10, ws11, ls1, ls2, ls3, ls4, ls5, ls6, ls7, ls8, ls9, ls10, ls11 are 10, 10, -10, -10, -5, -5, -2.5, -2.5, 2.5, 2.5, 5, 5, 10, -7.8, -22.8, -22.8, -7.8, -7.8, $17.8,-17.8,-7.8,-7.8,-17.8,-17,8,-7.8$ and $-7.8 m m$ respectively.

Co-ordinates $3^{\text {rd: }}$

Dig rectangular hole at $\mathrm{X}=0 ; \mathrm{y}=0$

$\mathrm{L}=5 \mathrm{~mm}, \mathrm{w}=2.5$

Co-ordinates $4^{\text {th }}$ :

Draw a rectangular at $\mathrm{X}=0, \mathrm{Y}=0$

(Rotation angle 45) $\mathrm{l}=5 \mathrm{~mm}, \mathrm{w}=5 \mathrm{~mm}$

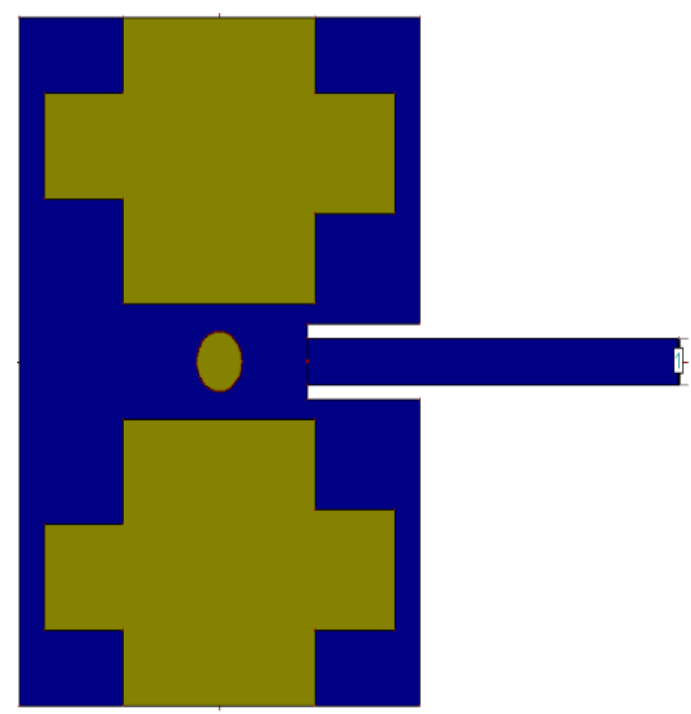

figure 2(a): Rectangular Microstrip Patch Antenna 


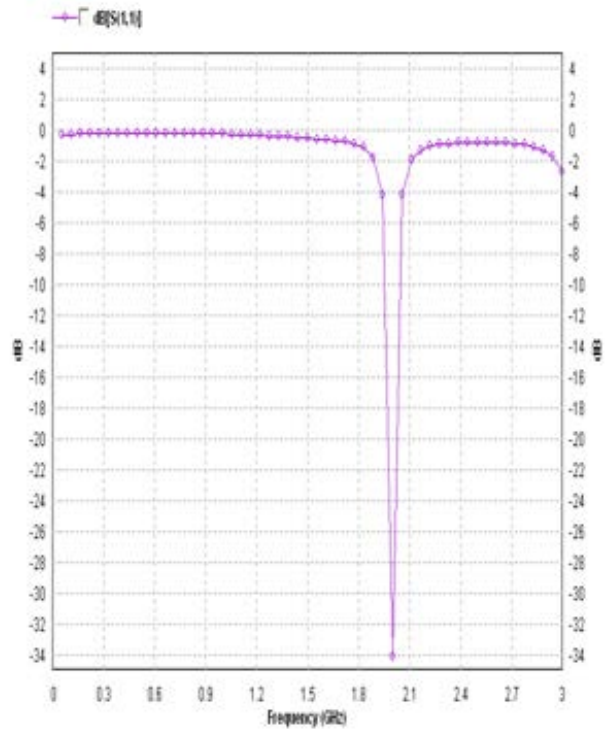

Figure2(b): Simulation Results of Return Loss of Rectangular Microstrip Patch Antenna

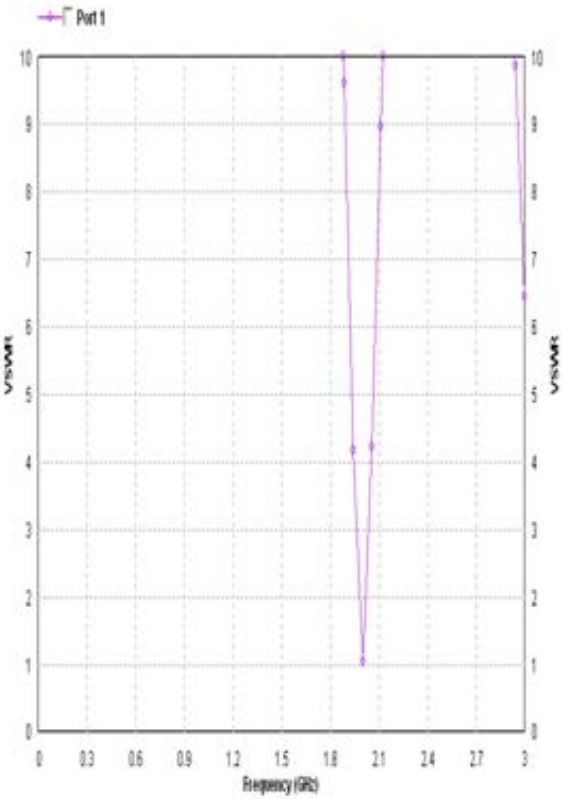

Figure 2(C): VSWR for microstrip Patch Antenna

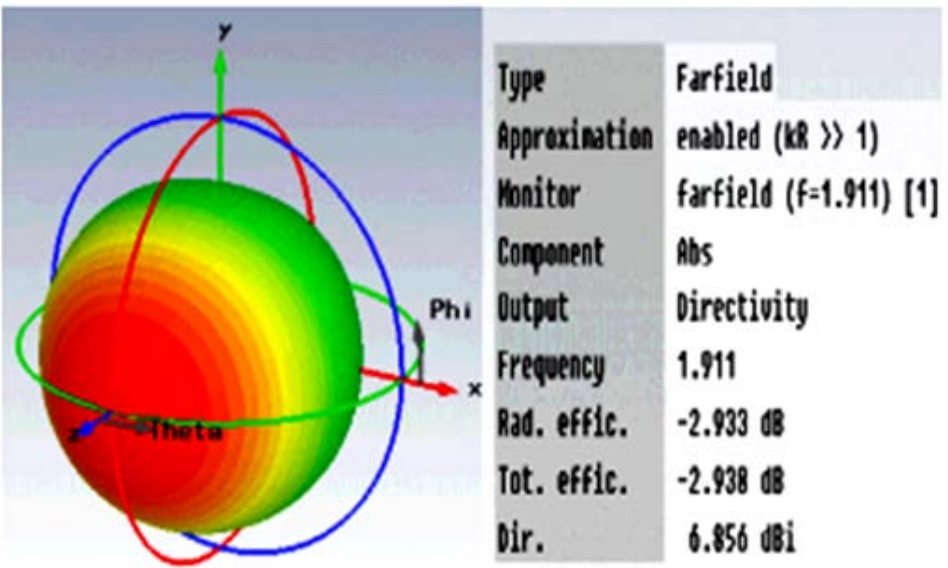




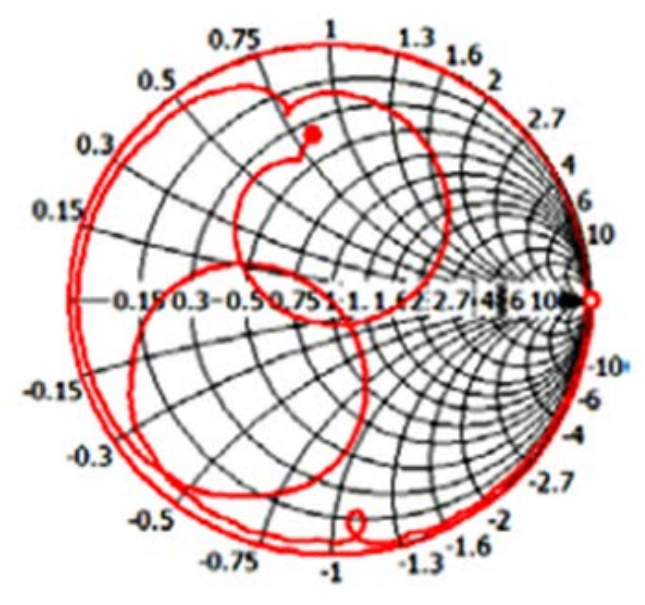

Fig2 (e) Smith chart for modified microstrip patch antenna 1

\section{Conclusion}

This Paper describes the result of proposed design of different modified Rectangular microstrip patch antenna using FR4 were computed mathematically replicated on IE3D software. In this article, it is discovered that using FR4 Structure on a Rectangular Microstrip Patch Antenna at 1.60mm or 3.20mm Layer reduces Return Loss, VSWR, and increases Bandwidth.

The simulated result of modified rectangular microstrip patch antenna with FR4 structure are depicted Figure2(a) and 2(b)At 2.0GHz frequency simulated, whenever it is designed with FR4 structure at $3.20 \mathrm{~mm}$ it depicts return loss is $-33 \mathrm{~dB}$ and VSWR is 1.040 , which depicts a considerable diminution of return loss and VSWR using FR4 structure.

\section{References}

[1]Dheeraj Bhardwaj, Komal Sharma’' Dual band and Broadband Rectangular Patch Mircostrip Antenna with T Shaped Slot for WiMax Application'’ International Journal of Engineering Research and Development ,Volume 3, Issue 12,September 2012.

[2] Bimal garg, Himanshu Srivastava, Prem Kumar, "Micro-strip Patch Antenna with Parameters Improvement Using "Symmetric Cylinder Shapes of Zero \& Four Segments" Meta-material Structure", International Journal of Computer Networks and Wireless Communications (IJCNWC), ISSN: 2250-3501, Vol.2, No.3, June 2012.

[3] Ravishankar Tiwari,' ' 't' shape microstrip patch antenna at 2.45ghz', Journal of Research in Electrical and Electronics Engineering (ISTP-JREEE),2013.

[4] F. Zhao, K. Xiao, W.-J. Feng, S.-L. Chai, and J.-J. Mao, "Design and Manufacture of the WideBand Aperture-Coupled Stacked Microstrip Antenna”, Progress in Electromagnetics Research C, Vol.7, Pg No. 37-50, 2009. 
[5] Yoshiaki Kamiya,Wataru CHUJO and Masayuki FUJISE "Design for Dual Frequency Microstrip Antenna using Annular Slot Aperture Coupling.” Antenna and Propagation Society International Symposium, 1991.

[6] S.M.Duffy and D.M.Pozar "Circularly Polarized Aperture Coupled Antenna” electronics letters Vol. 31 No. 16, 3rd August 2002.

[7] Rashid. A Saeed, S.Khatun, Borhauddin, M.A. Khazani, Raina A Mokhtar "Design of Single Fed Aperture coupled Microstrip antenna for WLAN".IEEE Transaction on Antenna and Propagation, 2005.

[8] Manoj Singh, Ananjun Basu and S.K.Koul "Design of Aperture Coupled Fed Microstrip Antenna for Wireless Communication”/ IEEE , Indian Conference, Pg No: 1-5, 2006.

[9] M.S.R. Mohd Shah, M.K.Suaidi, M.Z.A.Abdul Aziz, M.F.Abd Kadir, M.K.A. Rahim "Dual Polarization Microstrip Patch Array Antenna for WLAN Applications"/ Antennas and Propagation, 2009. EuCAP 2009. 3rd European Conference, 2009.

[10] V.G.Veselago"The electrodynamics of substances with simultaneously negative value $\varepsilon$ and $\mu$ " Sov. Phys. Uspekekhy.10 (4 ), pp 509-514, 1968.

[11] W.L. Stutzman, G.A. Thiele, Antenna Theory and design, John Wiley \& Sons, 2nd Ed., New York, 1998. 